\title{
Mid-infrared spectra of cometary dust: the evasion of its silicate mineralogy
}

\author{
H. Kimura, T. Chigai, and T. Yamamoto
}

\author{
Institute of Low Temperature Science, Hokkaido University, Kita-ku Kita-19 Nishi-8, Sapporo 060-0819, Japan \\ e-mail: hiroshi_kimura@lowtem.hokudai.ac.jp
}

Received 2 October 2007 / Accepted 5 February 2008

\begin{abstract}
Infrared spectra of dust in cometary comae provide a way to identify its silicate constituents, and this is crucial for correctly understanding the condition under which our planetary system is formed. Recent studies assign a newly detected peak at a wavelength of $9.3 \mu \mathrm{m}$ to pyroxenes and regard them as the most abundant silicate minerals in comets. Here we dispense with this pyroxene hypothesis to numerically reproduce the infrared features of cometary dust in the framework of our interstellar dust models. Presolar interstellar dust in a comet is modeled as fluffy aggregates consisting of submicrometer-sized organic grains with an amorphous-silicate core that undergoes nonthermal crystallization in a coma. We assert that forsterite $\left(\mathrm{Mg}_{2} \mathrm{SiO}_{4}\right)$ is the carrier of all the observed features, including the $9.3 \mu \mathrm{m}$ peak and that the major phase of iron is sulfides rather than iron-rich silicates.
\end{abstract}

Key words. comets: general - meteors, meteoroids - solar system: formation - dust, extinction - infrared: solar system

\section{Introduction}

Comets formed as an agglomeration of protosolar nebular condensates and/or presolar interstellar dust. Regardless of the environment in which the agglomeration took place, silicate is one of the most abundant constituents of cometary matter. Furthermore, mineralogy of the silicate is a key to the environment of comet formation (e.g., Nuth III \& Johnson 2006). If the nebular condensates predominate, silicates in cometary dust would consist not only of forsterite $\left(\mathrm{Mg}_{2} \mathrm{SiO}_{4}\right)$, but also of enstatite $\left(\mathrm{MgSiO}_{3}\right)$ (Lodders 2003). If interstellar dust is the primary component, amorphous silicates are in the majority because there are no crystalline silicates in the interstellar medium (Greenberg 1982; Kemper et al. 2004).

Spectroscopic infrared observations of thermal emission from cometary dust have revealed sharp peaks that are attributed to olivine (Campins \& Ryan 1989). Recent discoveries of a $9.3 \mu \mathrm{m}$ peak shed light on the presence of pyroxenes. Magnesium-rich pyroxene is said to produce this peak because it is the most abundant mineral in the dust of comets C/1995 O1 (Hale-Bopp) and C/2001 Q4 (NEAT) (Wooden et al. 1999, 2004; Harker et al. 2002; Hayward et al. 2000); Lisse et al. (2006) interpret the 9.3 micron feature in the infrared spectra for ejecta of comet 9P/Tempel 1 produced by NASA's Deep Impact mission with an abundance of iron-rich pyroxenes in the ejecta, while Harker et al. (2007) attribute the same feature to magnisium-rich pyroxenes.

Silicate minerals are commonly identified by a comparison between the positions of peaks in spectroscopic observations of cometary comae and those in laboratory measurements of absorption spectra for synthetic minerals. This identification method may mislead us about the silicate mineralogy of cometary dust, because the positions of peaks in the infrared spectra of small particles depend not only on the composition, but also on the internal and external structures of the particles (Bohren \& Huffman 1983). In this paper, we reconsider the origin of observed mid-infrared features by computing the infrared spectra of cometary dust based on our model of interstellar dust.

\section{Model descriptions and computational methods}

We consider incorporation of interstellar dust in a comet nucleus and nonthermal crystallization of its amorphous silicate component within a coma. Interstellar dust is modeled as fluffy aggregates of submicron grains having an organic refractory mantle and an amorphous silicate core (Kimura et al. 2003a). A cluster of submicron grains with amorphous silicate enclosed within carbonaceous material is common in primitive interplanetary dust particles of supposedly cometary origin, which were collected in the stratosphere (Keller et al. 1996, 2000). The model is also consistent with the impact ionization mass spectra of dust in the coma of comet 1P/Halley, suggesting that a chondritic core is covered by refractory organic material (Kissel \& Krueger 1987; Jessberger 1999). The elemental abundances of interstellar dust manifest the mass ratio close to unity between organic refractory and amorphous silicate in the dust (Kimura et al. 2003a). This also agrees with the elemental abundances of cometary dust and subsequently with the mass ratio of organic-to-silicate materials in cometary dust (see Jessberger et al. 1988). In spite of their presence in comet nuclei, we do not consider ices that typically sublimate too quickly to contribute to observed infrared spectra of cometary comae.

Once the dust is heated up above a few hundred kelvins in a coma, chemical reactions in the organic refractory mantle begin to heat the amorphous silicate core (Yamamoto \& Chigai 2005). As a consequence of the heat flow, the amorphous silicate core partially crystallizes to form a forsterite layer on the core surface. Experiments proved that this nonthermal crystallization works at room temperature on simulated submicron grains (Kaito et al. 2006, 2007). 


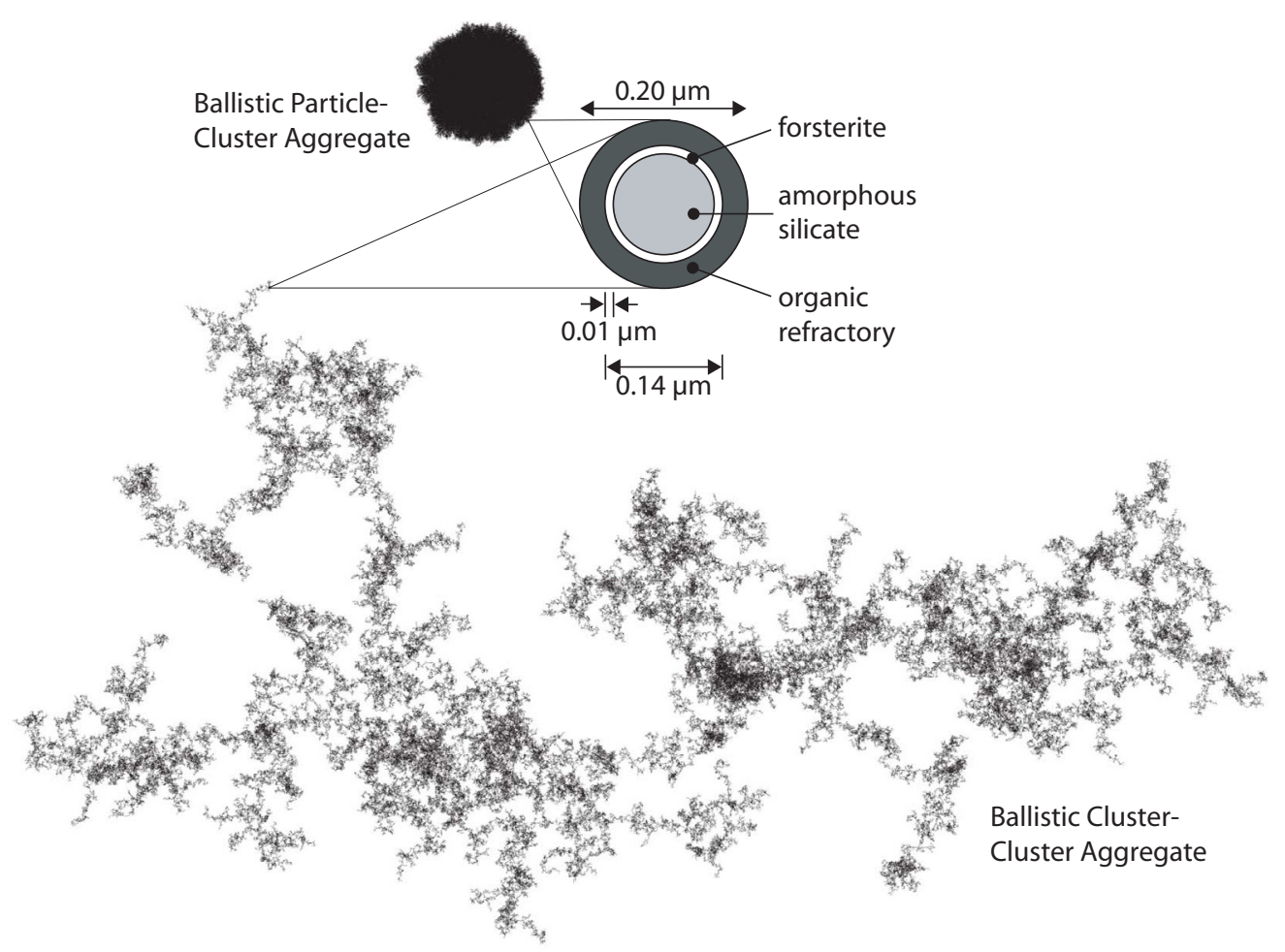

Fig. 1. A graphic illustration of dust aggregates consisting of $2^{20}$ submicron grains in a cometary coma, together with an enlarged picture of the constituent grains. Each grain has the layered structure of an amorphous silicate core, a forsterite inner mantle, and an organic refractory outer mantle.

To represent high and low degrees of structural changes, we use ballistic particle-cluster and cluster-cluster aggregates formed by hit-and-stick collisions (Fig. 1). All the observed characteristics of light scattering by cometary dust are reproduced well by such fluffy aggregates if the radii of their constituent grains (monomers) are $a_{\mathrm{m}}=0.1 \mu \mathrm{m}$ (Kimura et al. 2003b, 2006; Kolokolova et al. 2007). This monomer size results in the radius of the core being $a_{\mathrm{si}}=0.07 \mu \mathrm{m}$ and the thickness of the mantle $h_{\text {or }}=0.03 \mu \mathrm{m}$ (Kimura et al. 2003a). The surface of the core is forsterite over the thickness $h_{\mathrm{fo}}=0.01 \mu \mathrm{m}$, while the inner region of the core remains amorphous (see Fig. 1). As a typical size of dust aggregates in a coma, we take $a_{\mathrm{V}}=10 \mu \mathrm{m}$ (i.e., $2^{20}$ monomers) where $a_{\mathrm{V}}$ is the radius of spheres having equal volumes.

Our computational technique is an extension of the instructions supplied by Mukai et al. (1992) and Okamoto et al. (1994) to calculate the absorption cross section of an aggregate with given refractive indices of the monomers. The average refractive indices of monomers are derived from an effective medium approximation for three-layered spherical grains (Voshchinnikov $\&$ Mathis 1999). We take the refractive indices of organic refractory, crystalline $\mathrm{Mg}_{2} \mathrm{SiO}_{4}$, and amorphous $\mathrm{Mg}_{2} \mathrm{SiO}_{4}$ from $\mathrm{Li}$ \& Greenberg (1997), Sogawa et al. (2006), and Scott \& Duley (1996), respectively.

\section{Results and discussion}

Figure 2 shows our numerical results on the infrared spectra of cometary dust in the wavelength range from 8 to $13 \mu \mathrm{m}$. Vertical shaded bars indicate the ranges of wavelengths where infrared features were observed for several comets (Hanner et al. 1997; Wooden et al. 2004). Although the spectral shapes of infrared features vary from one comet to another and depend on the observational epoch, positions of the features are independent of comet characteristics. Therefore, our investigation will focus on the positions of infrared peaks and thus perfect fits to various spectral shapes go beyond the scope of this paper. The infrared spectra are here simulated with a spectral variation in the absorption cross section that is normalized to unity at a wavelength of $8 \mu \mathrm{m}$. The model spectra demonstrate five distinct peaks at wavelengths of $9.3,10.1,10.4,11.3$, and $11.9 \mu \mathrm{m}$. The positions of the peaks are well within the wavelength ranges of the observed infrared features indicated by vertical shaded bars. Moreover, major peaks located at 10.1 and $11.3 \mu \mathrm{m}$ are comparable in strengths, as expected from infrared observations of cometary dust (see, e.g., Hanner et al. 1999).

Although we do not play with model input parameters, we note that the positions of the peaks rarely change with the parameters. Any other assumptions about the size do not make any difference in the absorption cross section per volume for ballistic cluster-cluster aggregates (cf. Okamoto et al. 1994; Kolokolova et al. 2007). Furthermore, the absorption cross section per volume is almost independent of grain configuration for small aggregates with $a_{\mathrm{V}} \leq 10 \mu \mathrm{m}$ (Kolokolova et al. 2007). For large ballistic particle-cluster aggregates with $a_{\mathrm{V}} \gg 10 \mu \mathrm{m}$, infrared features tend to disappear, but the positions of the features stay nearly at the same wavelengths (see Okamoto et al. 1994). As long as small constituent grains $\left(a_{\mathrm{m}}<1 \mu \mathrm{m}\right)$ are concerned, the positions of the peaks are determined by the volume fraction of each material, but not by the grain size. Therefore, our results on the positions of infrared features are insensitive to the model parameters, if the mass ratio of organic refractory to silicate is kept the same.

All the crystal features in our calculated spectra are associated solely with forsterite, since no other crystals are involved in the calculation. We can identify forsterite even with the $9.3 \mu \mathrm{m}$ peak, although it has been recognized as a pyroxene feature. Note that all crystal features of comet C/1995 O1 (Hale-Bopp) 


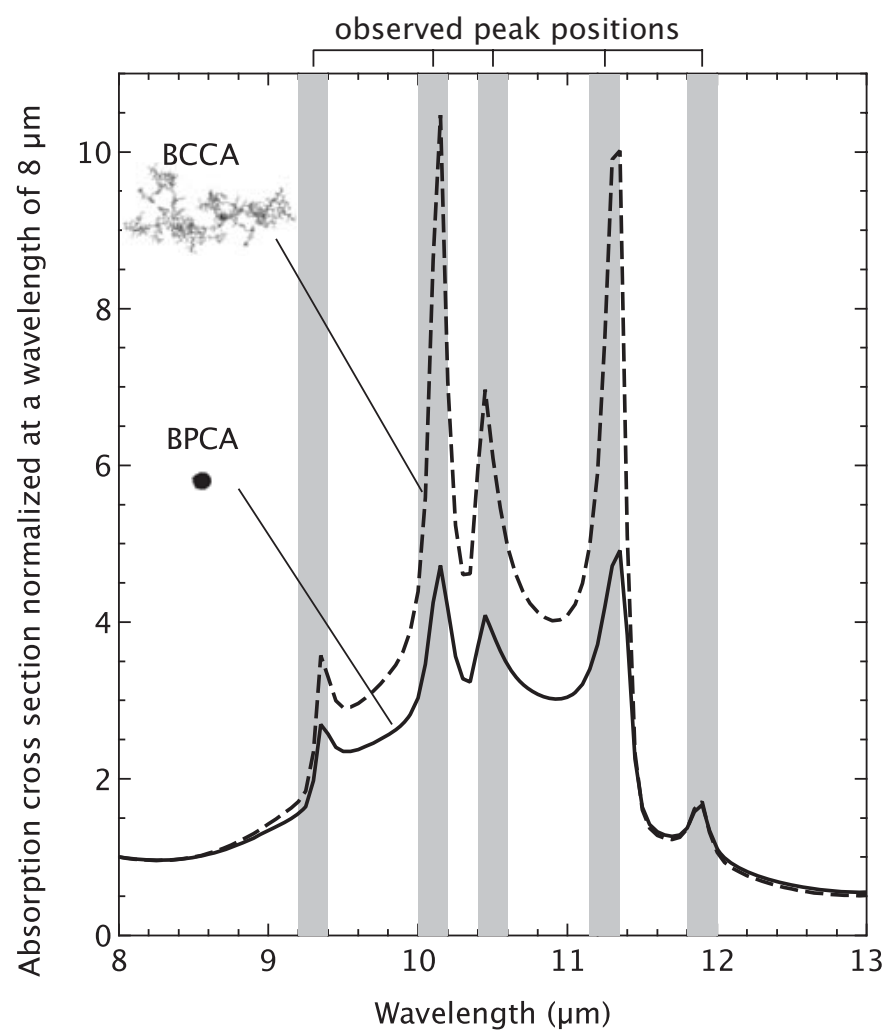

Fig. 2. Infrared spectra of ballistic particle-cluster aggregates (BPCAs) and ballistic cluster-cluster aggregates (BCCAs) consisting of $2^{20}$ submicron grains in a cometary coma. The spectrum is represented by the spectral variation in the absorption cross section normalized at a wavelength of $8 \mu \mathrm{m}$. Vertical shaded bars indicate the positions of infrared features observed for cometary comae (Hanner et al. 1997; Wooden et al. 2004).

observed with the Infrared Space Observatory at longer wavelengths of $16,27.5,19.5,23.5,27.5$, and $33.5 \mu \mathrm{m}$ were attributed to forsterite (Crovisier et al. 1997). We confirmed this identification by performing further computations up to a wavelength of $60 \mu \mathrm{m}$ as an extension of this study. In conclusion, there is no need to invoke the presence of pyroxenes for all the thermal emission features of cometary dust.

Our model of presolar interstellar dust successfully reproduces all the emission peaks around $10 \mu \mathrm{m}$ observed for cometary dust. It is worth noting that the absorption spectra for homogeneous spheres or ellipsoids of forsterite do not show a peak at $9.3 \mu \mathrm{m}$ (Sogawa et al. 2006). Therefore, the success of our model in reproducing all the mid-infrared peaks comes from the layered structure of monomers where an organic outer mantle and a forsteritic inner mantle encase an amorphous silicate core. This clearly shows that identifying minerals through the infrared spectra is more complex than previously considered. Moreover, our model indicates that the presence of crystalline silicates inside comae of comets does not preclude a predominance of presolar interstellar dust in their nuclei.

In contrast to our model, infrared spectra of solid materials released from comets 9P/Tempel 1 and C/1995 O1 (Hale-Bopp) were explained by the predominance of ferrosilite (iron-rich pyroxene) in the former and the predominance of magnesium-rich pyroxenes in the latter. These interpretations are clearly at odds with the similarity in the infrared spectra between 9P/Tempel 1 and C/1995 O1. From an analysis of the mass spectra measured in situ for dust in the coma of comet 1P/Halley, Jessberger et al. (1988) revealed that silicates in the dust are magnesium-rich, which was taken as evidence for a low-temperature history of the dust. Furthermore, their common-factor analysis of the elements in Halley's dust suggests that an abundance of iron in cometary dust is in the form of sulfides. These results for $1 \mathrm{P} /$ Halley dust are entirely consistent with the recent analyses of 81P/Wild 2 dust samples, in which silicates are magnesium-rich and the major phase of iron is sulfides (see, Zolensky et al. 2006). Cometary dust composed of magnesium-rich silicates and iron-sulfides is consistent with our model of cometary dust, because iron sulfides do not show any features in the $10 \mu \mathrm{m}$ region. We conclude that a predominance of iron-rich silicate minerals in cometary dust is highly unlikely, contrary to the recent identification of ferrosilite.

Acknowledgements. We would like to thank Elmar K. Jessberger for fruitful discussions and an anonymous referee for useful suggestions. This work is supported by the Ministry of Education, Culture, Sports, Science, and Technology MEXT (Monbu Kagaku Sho) under Grant-in-Aid for Scientific Research on Priority Areas.

\section{References}

Bohren, C. F., \& Huffman, D. R. 1983, Absorption and Scattering of Light by Small Particles (New York: Wiley-Interscience)

Campins, H., \& Ryan, E. V. 1989, ApJ, 341, 1059

Crovisier, J., Leech, K., Bockelée-Morvan, D., et al. 1997, Science, 275, 1904

Greenberg, J. M. 1982, in Comets, ed. L. L. Wilkening (Tucson: Univ. Arizona Press), 131

Hanner, M. S., Gehrz, R. D., Harker, D. E., et al. 1997, Earth, Moon, Planets, 79, 247

Hanner, M. S. 1999, Space Sci. Rev., 90, 99

Harker, D. E., Wooden, D. H., Woodward, C. E., \& Lisse, C. M. 2002, ApJ, 580, 579; Erratum: 2004, ApJ, 615, 1081

Harker, D. E., Woodward, C. E., Wooden, D. H., Fisher, R. S., \& Trujillo, C. A. 2007, Icarus, 190, 432

Hayward, T. L., Hanner, M. S., \& Sekanina, Z. 2000, ApJ, 538, 428

Jessberger, E. K. 1999, Space Sci. Rev., 90, 91

Jessberger, E. K., Christoforidis, A., \& Kissel, J. 1988, Nature, 332, 691

Kaito, C., Sasaki, S., Miyazaki, Y., et al. 2006, Adv. Geosci., 7, 125

Kaito, C., Miyazaki, Y., Kumamoto, A., \& Kimura, Y., 2007, ApJ, 666, L57

Keller, L. P., Thomas, K. L., \& McKay, D. S. 1996, Lunar Planet. Sci., 27, 659

Keller, L. P., Messenger, S., \& Bradley, J. P. 2000, J. Geophys. Res., 105, 10397

Kemper, F., Vriend, W. J., \& Tielens, A. G. G. M. 2004, ApJ, 609, 826

Kimura, H., Mann, I., \& Jessberger, E. K. 2003a, ApJ, 583, 314

Kimura, H., Kolokolova, L., \& Mann, I. 2003b, A\&A, 407, L5

Kimura, H., Kolokolova, L., \& Mann, I. 2006, A\&A, 449, 1243

Kissel, J., \& Krueger, F. R. 1987, Nature, 326, 755

Kolokolova, L., Kimura, H., Kiselev, N., \& Rosenbush, V. 2007, A\&A, 463, 1189

Li, A., \& Greenberg, J. M. 1997, A\&A, 323, 566

Lisse, C. M., VanCleve, J., Adams, A. C. et al. 2006, Science, 313, 635

Lodders, K. 2003, ApJ, 591, 1220

Mukai, T., Ishimoto, H., Kozasa, T., Blum, J., \& Greenberg, J. M. 1992, A\&A, 262,315

Nuth III, J. A., \& Johnson, N. M. 2006, Icarus, 180, 243

Okamoto, H., Mukai, T., \& Kozasa, T. 1994, Planet. Space Sci., 42, 643

Scott, A., \& Duley, W. W. 1996, ApJS, 105, 401

Sogawa, H., Koike, C., Chihara, H., et al. 2006, A\&A, 451, 357

Voshchinnikov, N. V., \& Mathis, J. S. 1999, ApJ, 526, 257

Wooden, D. H., Harker, D. E., Woodward, C. E., et al. 1999, ApJ, 517, 1034

Wooden, D. H., Woodward, C. E., \& Harker, D. E. 2004, ApJ, 612, L77

Yamamoto, T., \& Chigai, T. 2005, in Highlights of Astronomy, ed. O. Engvold (San Francisco: ASP), 522

Zolensky, M. E., Zega, T. J., Yano, H., et al. 2006, Science, 314, 1735 